\title{
Editorial: pituitary tumors
}

\author{
Marvin Bergsneider • Anthony P. Heaney • \\ John Y. K. Lee
}

Published online: 15 April 2014

(c) Springer Science+Business Media New York 2014

The management of pituitary adenomas is evolving. Three decades ago, diagnostic modalities were relatively crude and surgery generally the only option for treatment. Today, we are able to image 2-mm microadenomas using special MRI sequencing. Surgery has been supplanted as the upfront treatment for prolactinomas by dopamine agonist therapy, and some may argue that the efficacy of medical treatment of acromegaly may soon surpass surgical removal of growth hormone secreting pituitary adenomas. Other treatment modalities, such as stereotactic radiosurgery, have gained a stronger foothold in the management paradigm. The surgical management of pituitary adenomas has also undergone an evolutionary, perhaps revolutionary, change. The endoscopic endonasal approach has expanded

\section{Bergsneider $(\square)$}

Department of Neurosurgery, David Geffen School of Medicine at UCLA, Gonda 3357, BOX 951761, Los Angeles, CA 90095-1761, USA

e-mail: mbergsneider@mednet.ucla.edu

\author{
A. P. Heaney \\ Division of Endocrinology, Department of Medicine, David \\ Geffen School of Medicine at UCLA, 24-130 WH, \\ BOX 957073, Los Angeles, CA 90095-7073, USA \\ J. Y. K. Lee \\ Department of Neurosurgery, University of Pennsylvania, \\ Washington Square West Building 235 S. 8th Street, \\ Philadelphia, PA 19106, USA
}

the surgeon's ability to remove invasive and/or larger tumors that formerly were considered inoperable, required a craniotomy, or were relegated to "partial surgical removal."

The clinical manifestations of pituitary adenomas can be debilitating and even lethal. Depending on the size and hormonal activity of the tumor, patients can present symptomatology ranging from subtle loss of red color perception to complete blindness; from male loss of libido to panhypopituitarism; from subtle changes in hand size to 8 feet tall giants with short life expectancies; or from morbid obesity to overt psychosis. These tumors are located deep at the base of the skull, in close proximity and/or overt involvement of critical neurovascular structures. No other tumor mandates such a wide range of interdisciplinary specialists who must work cohesively as a team to provide optimal care to the patient. The traditional treating duo of a neurosurgeon and endocrinologist has expanded to regularly include endoscopic head and neck skullbase specialists, interventional neuroradiologists, and radiation oncologists. More recently, even the neuro-oncologist is asked to join the team for the management of highly aggressive and cancerous lesions.

In this special issue, we have solicited leading authorities in their respective fields to provide the most up-to-date information on key areas of management and controversy within the field. We have also included several original research papers related to pituitary tumors. We hope this special issue can serve as a readily available resource for a wide variety of health care professionals caring for pituitary tumor patients. As the diagnosis and management of pituitary tumors becomes more complex and subspecialized, it is all more important that all physicians treating these patients have a strong medical knowledge foundation to best assist in the care. 


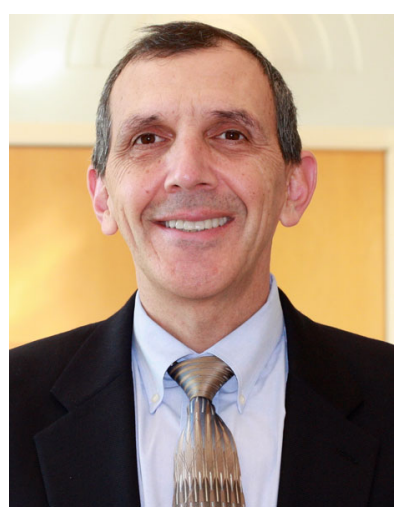

\section{Marvin Bergsneider}

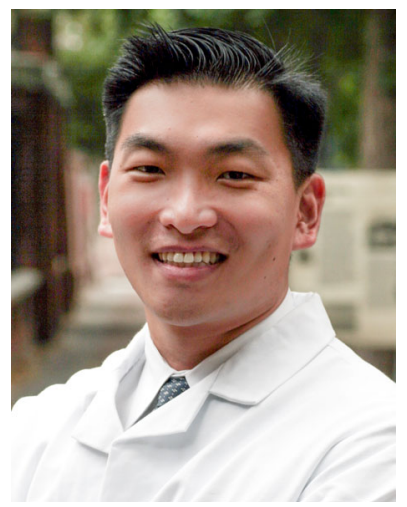

John Y. K. Lee

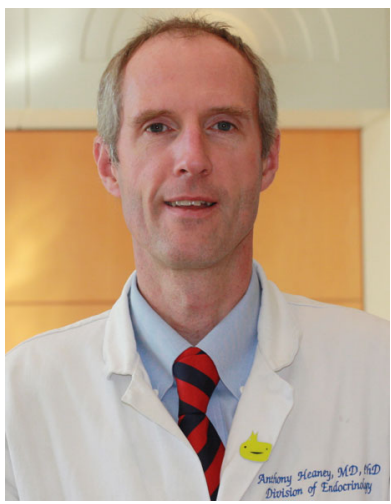

Anthony P. Heaney 